\title{
La laïcité devant le Conseil d'État en 2018
}

\section{Pierre-Henri Prélot}

\section{OpenEdition}

\section{Journals}

Édition électronique

URL : http://journals.openedition.org/rdr/307

DOI $: 10.4000 /$ rdr.307

ISSN : 2534-7462

\section{Éditeur}

Presses universitaires de Strasbourg

\section{Édition imprimée}

Date de publication : 1 mai 2019

Pagination : 209-220

ISBN : 979-10-344-0045-4

ISSN : 2493-8637

\section{Référence électronique}

Pierre-Henri Prélot, « La laïcité devant le Conseil d'État en 2018 », Revue du droit des religions [En ligne], 7 | 2019, mis en ligne le 09 octobre 2019, consulté le 19 novembre 2020. URL : http:// journals.openedition.org/rdr/307 ; DOI : https://doi.org/10.4000/rdr.307

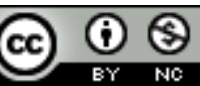

La revue du droit des religions est mise à disposition selon les termes de la Creative Commons Attribution - Pas d'Utilisation Commerciale 4.0 International - CC BY-NC 4.0. 


\section{LA LAÏCITÉDEVANT LECONSELLD'ÉTAT EN 2018}

\section{Pierre-Henri PRÉLOT}

Université de Cergy-Pontoise

$\mathrm{L}$ e Conseil d'État a rendu en 2018 deux décisions intéressant le droit des religions et de la laïcité. La première avait pour objet une question prioritaire de constitutionnalité (QPC) visant à invalider l'élection d'un professeur de la faculté de théologie catholique, par ailleurs prêtre de son état, en qualité de président de l'université de Strasbourg ${ }^{1}$. La seconde portait sur la légalité du décret du 3 mai 2017 relatif aux aumôniers militaires, hospitaliers et pénitentiaires et à leur formation civile et civique, ainsi que l'arrêté interministériel subséquent du 5 mai $2017^{2}$. Les deux recours ont été rejetés sans grande surprise dans deux décisions rendues le même jour, le 27 juin 2018, mais par deux formations de jugement différentes. Si ces deux décisions, outre leur coïncidence de date, méritent un examen simultané, c'est parce qu'elles sont révélatrices des tensions que suscitent aujourd'hui le principe de laïcité et la place des religions dans l'espace social, tensions dont l'Église catholique a cessé d'être l'épicentre même s'il se trouve que les deux affaires la concernent au premier chef.

Dans le litige relatif à l'élection d'un prêtre catholique à la présidence de l'université de Strasbourg, la question posée n'était pas totalement nouvelle. L'accès des ministres du culte à l'enseignement supérieur a été validé dans son principe, il y a plus d'un siècle, par le commissaire du gouvernement

1. CE, 27 juin $2018,4^{\text {e }}$ et $1^{\text {re }}$ ch., $n^{\circ} 419595$, SNESUP.

2. CE, 27 juin 2018, $2^{\mathrm{e}}$ et $7^{\mathrm{e}} \mathrm{ch} ., \mathrm{n}^{\circ} 412039$, Union des associations diocésaines de France et $\mathrm{M}^{\text {gr }}$ Vingt-Trois, archevêque de Paris. 
Helbronner dans ses conclusions à l'arrêt Bouteyre ${ }^{3}$, et la présidence de son université fait partie des multiples responsabilités qu'un professeur de l'enseignement supérieur peut être amené à endosser dans le cours de sa carrière. Sans doute la solution demandait-elle à être confirmée puisque jamais à ce jour l'occasion ne s'était présentée de saisir le juge en bonne et due forme. Par ailleurs, l'entrée en vigueur de la question prioritaire de constitutionnalité (QPC), autorisant les requérants à solliciter l'intervention du Conseil constitutionnel dans le litige, était de nature à renouveler quelque peu les termes du débat. Mais au-delà de ces considérations ponctuelles, évidemment importantes, cette affaire s'inscrit dans un contexte plus général de remise en cause de toute forme de présence religieuse au sein de l'université, et c'est ce qui vient donner à la résurgence de ce vieux débat séculaire toute son actualité. Le colloque de la Conférence des présidents d'université (CPU) consacré en 2003 à «la laïcité à l'Université», a ouvert un débat sur la nécessité de renforcer la laïcité dans cet espace de liberté intellectuelle que doit être l'alma mater ${ }^{4}$, et les revendications en ce sens se sont multipliées depuis. En particulier, l'extension aux universités publiques de l'interdiction $\mathrm{du}$ port des signes religieux ostensibles, au risque d'une secondarisation accrue de l'enseignement supérieur, suscite régulièrement des propositions de loi de la part de parlementaires que la question obsède. Dans ces conditions le rappel, par le Conseil d'État, de la tradition libérale de l'Université, prend une signification singulière qui relègue au second plan le vieil anticléricalisme dont cette affaire n'est pas complètement exempte.

Dans le recours exercé contre le décret et l'arrêté relatifs aux aumôniers militaires, hospitaliers et pénitentiaires, c'est l'obligation faite aux futurs aumôniers de posséder un diplôme de formation civile et civique, couramment dénommé «DU laiicité», qui était contestée par l'Union des associations diocésaines de France et par l'archevêque de Paris. La présence d'aumôniers catholiques est de tradition immémoriale dans ces institutions où se joue le salut des âmes que sont l'hôpital, la prison, les enceintes militaires et les champs de bataille, et jamais il n'avait été question jusqu'ici d'exiger des aumôniers qu'ils possédassent des connaissances autres que celles qui leur permettent d'accomplir leur office religieux. La situation serait d'ailleurs restée inchangée sans la volonté des pouvoirs publics d'encourager la nomination d'aumôniers musulmans dans une finalité d'intégration républicaine de l'islam et de lutte contre la radicalisation religieuse. Le gouvernement

3. CE, 10 mai 1912, Abbé Bouteyre: GAJA 2018, nº 24.

4. Sur cette question on se permet de renvoyer au dossier consacré par l'AJDA à la laïcité en 2017, et en particulier à notre contribution sur «L'université publique et la laïcité », AJDA 2017, p. 1375-1380. 
dirigé par Bernard Cazeneuve a ainsi mis en place en 2017 une formation spécifique, dispensée par les universités publiques et des établissements privés d'enseignement général (instituts catholiques en particulier), qui comprend notamment des enseignements relatifs aux institutions de la République et à la laïcité, aux grands principes du droit des cultes ainsi qu'aux sciences humaines et sociales des religions. L'impossibilité qu'il y a, dans un régime de laicité prohibant toute forme de traitement différencié des religions, d'imposer la possession du diplôme laïcité sanctionnant le suivi de cette formation aux seuls aumôniers musulmans, a donc conduit les pouvoirs publics à en faire une obligation générale. En bonne logique c'est l'ordre public, dont l'article 10 de la déclaration de 1789 (DDHC) et l'article $1^{\text {er }}$ de la loi de 1905 ont fait le butoir où vient s'arrêter la liberté de religion, qui sert une fois encore de support à cette réglementation que le Conseil d'État a entérinée après examen de ce recours particulièrement circonstancié.

\section{L'APTITUDE D'UN MINISTRE DU CULTE À PRÉSIDER UNE UNIVERSITÉ PUBLIQUE}

La création au sein de l'université de Strasbourg d'une faculté de théologie catholique remonte à 1902. Issue d'une convention entre le gouvernement du Reich et le Saint-Siège, celle-ci relève aujourd'hui de cet ensemble protéiforme qui a pour nom le droit local. Hors de l'Alsace-Moselle et de ses règles propres, il n'existe plus en France d'enseignement public de la théologie. Mais quoiqu'il en soit de cette spécificité, la faculté de théologie catholique (comme au reste son homologue protestante), aujourd'hui intégrée à l'université en tant qu'institut (C. éduc., art. L. 713-9), relève pour l'essentiel du droit commun universitaire. C'est ainsi que les enseignants sont recrutés selon la procédure classique des comités de sélection, après qualification par une commission spéciale consultative assimilée à une section du CNU (section 76). Toutefois leur nomination, s'agissant d'enseignants appelés à dispenser des grades théologiques, requiert (article 3 de la convention) l'accord préalable de l'archevêque de Strasbourg.

L'élection en décembre 2016 d'un professeur de la faculté de théologie catholique, par ailleurs prêtre de son état, à la présidence de l'université de Strasbourg, ne relevait en aucune façon du droit local, que le syndicat requérant s'est d'ailleurs soigneusement gardé de mettre en cause ${ }^{5}$. Elle sou-

5. V. TA Strasbourg, 14 déc. 2017, $\mathrm{n}^{\circ}$ 1703016, SNES. Dans sa requête le SNES opposait nettement les fonctions de professeur des universités qui «ne sont pas, par elles-mêmes, 
levait des questions de droit universitaire général, et c'est en quoi la solution dégagée par l'arrêt du Conseil d'État intéresse le régime français de la laïcité qu'elle conforte sur un point où le droit jusqu'ici restait écrit en lettres filigranes, même si ses solutions ne faisaient guère de doute. Le tribunal administratif de Strasbourg ayant opposé aux requérants qui dénonçaient l'élection de Michel Deneken à la présidence de l'université les dispositions législatives du Code de l'éducation, il ne leur restait plus qu'à en contester la constitutionnalité devant la cour d'appel de Nancy par la voie d'une QPC, que cette dernière a aussitôt transmise au Conseil d'État. Lequel a considéré qu'il n'y avait pas lieu de déranger le Conseil constitutionnel pour une question qui, outre qu'elle n'est pas nouvelle, est dépourvue de tout caractère sérieux.

S'il n'y a rien de nouveau sous le soleil, explique le Conseil d'État, c'est parce que l'affaire en cause relève banalement du droit de la fonction publique générale, et qu'en application du principe de laïcité «l'accès aux fonctions publiques, dont l'accès aux fonctions de président d'université, s'effectue sans distinction de croyance et de religion». Avec la séparation des Églises et de l'État, les ministres du culte sont devenus des citoyens ordinaires et en cette qualité ils «sont également admissibles à toutes dignités, places et emplois publics, selon leur capacité, et sans autre distinction que celle de leurs vertus et de leurs talents» (art. 6 DDHC). Ce qui est en revanche interdit aux agents publics, c'est la «manifestation» de leurs croyances religieuses dans l'exercice de leurs fonctions, et notamment le port d'un signe ou d'une tenue marquant une appartenance religieuse. Comme tout agent public, une personne «élue aux fonctions de président d'université » reste en effet «tenue, eu égard à la neutralité des services publics qui découle également du principe de laicité, à ne pas manifester ses opinions religieuses dans l'exercice de ses fonctions ainsi qu'à un devoir de réserve en dehors de l'exercice de ces fonctions». Le Conseil d'État reprend ici la théorie des apparences qu'il avait érigée en critérium principal de la neutralité des agents publics dans son avis Delle Marteaux du 3 mai $2000^{6}$.

Il est significatif que dans cette affaire le Conseil d'État ait choisi de se fonder sur les solutions du droit de la fonction publique générale plutôt que sur les dispositions afférentes à la seule fonction enseignante. En procédant ainsi, il remet implicitement mais nécessairement en cause les interdictions

incompatibles avec un engagement religieux» et «les fonctions de direction» d'un président d'université qui «ne lui permettent pas de garantir la laïcité du service public de l'enseignement supérieur».

6. CE, avis, 3 mai 2000, n 217017, Delle Marteaux. 
énoncées tant au niveau législatif pour l'enseignement primaire (loi Goblet de 1886), qu'au niveau jurisprudentiel pour l'enseignement secondaire (jurisprudence Bouteyre), et dont la doctrine a souligné de longue date la fragilité 7 . On sait que dans ses conclusions à l'arrêt Abbé Bouteyre, le commissaire du gouvernement Helbronner avait construit son raisonnement en référence à l'enseignement supérieur où, compte tenu de la nature de l'enseignement et de la maturité des étudiants, rien ne s'opposait à ce que des ecclésiastiques pussent accéder aux fonctions d'enseignement ${ }^{8}$. Seulement, comme on l'a dit, la question posée dans le cas présent ne concernait pas l'accès aux fonctions d'enseignement, mais la possibilité pour un universitaire par ailleurs ecclésiastique de son état de présider son université. Si donc les raisonnements du conseiller d'État Helbronner indiquaient la solution du litige, ils étaient en revanche insuffisants à le résoudre, ainsi que l'a souligné le rapporteur Frédéric Dieu dans ses conclusions ${ }^{9}$.

Outre le principe de laïcité, le syndicat requérant invoquait le principe constitutionnel d'indépendance des enseignants-chercheurs. L'argument ici consistait à soutenir qu'un universitaire ayant la qualité de ministre du culte ne présenterait pas, compte tenu de son engagement religieux, les garanties d'indépendance que requièrent des fonctions de direction et de représentation d'une communauté universitaire dans son entier. Ainsi qu'on l'a souligné, la nomination des enseignants-chercheurs de la faculté de théologie catholique doit être approuvée par l'archevêque de Strasbourg en vertu de l'article 3 de la convention de 1902, et c'est à ce lien de dépendance religieuse que renvoyait implicitement la requête. Pour cette raison, soutenait le syndicat, les dispositions du Code de l'éducation relatives à l'élection des présidents d'université (L. 712-2) sont inconstitutionnelles «en ce qu'elles ne prévoient aucune incompatibilité entre la présidence d'un établissement d'enseignement et de recherche et l'exercice concomitant d'une charge ou d'une fonction

7. On renvoie ici au commentaire de l'arrêt Bouteyre aux Grands arrêts de la jurisprudence administrative.

8. «Pour l'enseignement supérieur public [...], la nature de l'enseignement donné, le caractère des personnes auxquelles il s'adresse, dispense en principe l'État de prendre (comme dans les autres ordres d'enseignement) la responsabilité des doctrines qui sont enseignées. Les élèves, les auditeurs, sont ici en âge de juger. L'enseignement qui leur est donné a pour but de développer la culture littéraire ou scientifique. L'incompatibilité entre cet enseignement, ou tout au moins certaines parties de cet enseignement, et l'état ecclésiastique n'a donc plus les mêmes raisons d'être. La foi religieuse, les dogmes des Églises s'accordent parfaitement avec le culte des lettres ou le goût des recherches scientifiques [...]»: Les grandes conclusions de la jurisprudence administrative 1831-1940, t. 1, Paris, LGDJ, 2015, n 51.

9. Nous le remercions d'avoir bien voulu nous transmettre ses conclusions qui ont beaucoup facilité la rédaction de ce commentaire. 
religieuse ${ }^{10} »$. Ici encore, l'argument de l'incompétence négative du législateur est balayé d'un revers de main par le Conseil d'État, au motif qu'un tel cumul de fonctions religieuses et universitaires et en soi «sans rapport avec les garanties qui s'attachent au respect du principe constitutionnel d'indépendance des enseignants-chercheurs ». De fait, en vertu d'une interprétation jurisprudentielle restrictive de ce principe constitutionnel d'indépendance progressivement transformé en peau de chagrin par la juridiction administrative, les garanties en cause se rapportent aujourd'hui exclusivement aux conditions de recrutement des universitaires ${ }^{11}$.

La Haute juridiction a donc considéré que l'engagement confessionnel, d'ordre privé, n'entraîne aucune capitis diminutio dans l'ordre académique ${ }^{12}$. Les enseignants-chercheurs des facultés de théologie de l'université de Strasbourg ne sont pas relégués dans le champ clos de leur enceinte facultaire. Ils ont vocation comme leurs collègues des autres disciplines à participer sans restriction à la vie de leur université. Bien évidemment, la solution libérale retenue par le Conseil d'État n'écarte absolument pas l'hypothèse d'un conflit de loyauté, les questions bioéthiques figurant un exemple topique des tensions susceptibles de survenir dans une université comprenant une faculté de médecine. Mais outre que l'affirmation du caractère laïque du service public de l'enseignement supérieur ${ }^{13}$ indique le sens dans lequel doit être résolu un éventuel conflit de valeurs, et bien que la loi de 2007 ait renforcé les pouvoirs des présidents, il reste dans les universités des instances de délibération collective qualifiées pour apaiser et si besoin résoudre de tels conflits de loyauté. De surcroît, l'énoncé classique selon lequel le président de l'université est tenu de «ne pas manifester ses opinions religieuses dans l'exercice de ses fonctions ainsi qu'à un devoir de réserve en dehors de l'exercice de ces fonctions» doit être compris en l'espèce comme une forme de mise en garde à l'égard du titulaire de la fonction.

10. CAA Nancy, ord. $\mathrm{n}^{\circ} 18 \mathrm{NC00333}$, SNES.

11. V. F. Melleray, "Que reste-t-il des "bases constitutionnelles du droit universitaire"? », in Les droits de l'Homme à la croisée des chemins. Mélanges en l'honneur de Frédéric Sudre, Paris, LexisNexis, 2018, p. 473-481.

12. On notera ici qu'avant d'en devenir le président, Michel Deneken avait été plusieurs années durant vice-président de son université, et que son état d'ecclésiastique n'avait jamais été contesté devant la justice.

13. C. éduc., art. L. 141-6. 


\section{L'EXIGENCE DE FORMATION À LA LAÏCITÉ DES AUMÔNIERS DES SERVICES PUBLICS}

L'intégration républicaine de l'islam, à laquelle travaillent depuis une vingtaine d'années les pouvoirs publics, requiert la mise en œuvre à son égard des dispositifs juridiques aménagés au profit des autres religions. Elle appelle en particulier l'installation d'aumôniers musulmans dans les services publics mentionnés à l'article 2 de la loi de $1905^{14}$. Cet accès s'est ouvert progressivement à l'hôpital, dans les prisons, mais également dans les armées avec l'institutionnalisation d'une aumônerie militaire à partir de $2005^{15}$. Toutefois la radicalisation d'une partie de la population musulmane, notamment chez les prisonniers, a fait prendre conscience de la nécessité qu'il y avait de sécuriser le recrutement des aumôniers, en leur imposant la possession d'un diplôme de formation civile et civique. L'idée d'une telle formation à la laïcité et aux principes républicains à l'intention des personnels religieux musulmans remonte aux années 2000, mais elle avait été massivement récusée au départ par les universités sollicitées à cette fin. La succession des attentats terroristes de 2015-2016 a fait évoluer les esprits, et l'engagement financier du ministère de l'Intérieur a permis de surmonter les dernières résistances du monde académique. La principale originalité du dispositif mis en place en 2017 tient à son caractère obligatoire, en ce qui concerne les aumôniers statutaires des armées, les aumôniers contractuels des hôpitaux, et les aumôniers rémunérés de l'administration pénitentiaire. Mais si ce dispositif vise à favoriser le recrutement des aumôniers musulmans, en revanche pour les cultes anciennement établis il impose à leurs futurs aumôniers des conditions nouvelles qui leur sont apparues aussi lourdes qu'inutiles. C'est ce qui a convaincu l'épiscopat catholique, et en particulier l'archevêque de Paris à l'origine d'un recours distinct, de contester devant le Conseil d'État le décret du 3 mai 2017 relatif aux aumôniers militaires, hospitaliers et pénitentiaires et à leur formation civile et civique, ainsi que l'arrêté interministériel du 5 mai 2017 relatif aux diplômes de formation civile et civique suivie par les aumôniers militaires d'active et les aumôniers hospitaliers et pénitentiaires et fixant les modalités d'établissement de la liste de ces formations.

14. L'article 2 de la loi de 1905 mentionne «les établissements publics tels que lycées, collèges, écoles, hospices, asiles et prisons».

15. À l'école en revanche c'est un mouvement inverse qui a prévalu, avec le gel de toute création d'aumônerie nouvelle. C'est pourquoi le décret attaqué ne mentionne pas l'aumônerie scolaire. 
À la lecture, le décret et l'arrêté attaqués sont d'un accès difficile, parce que le diplôme de formation civile et civique qu'ils mettent en place concerne trois institutions (armée, hôpitaux, prisons) dont les aumôniers relèvent de dispositifs juridiques jusqu'ici disparates, aucune réglementation statutaire commune à l'ensemble des aumôneries n'ayant jamais été édictée depuis la séparation. S'ils sont choisis par l'autorité religieuse dont ils relèvent, les aumôniers militaires et hospitaliers ont juridiquement un statut d'agents contractuels, alors que le recrutement des aumôniers des établissements pénitentiaires fait l'objet d'un agrément par le directeur interrégional des services pénitentiaires. À côté de ces trois catégories d'aumôniers rémunérés pour leurs interventions, les aumôniers qui interviennent à titre bénévole ne sont pas astreints à la possession du diplôme.

La compétence du pouvoir réglementaire pour organiser les conditions d'intervention des aumôniers au sein des services publics ne fait pas difficulté aux yeux du Conseil d'État. En ce qui concerne les aumôniers militaires et hospitaliers, elle repose en effet sur une habilitation législative expresse ${ }^{16}$. Quant aux aumôniers des prisons, les juges estiment que la compétence du pouvoir réglementaire résulte implicitement des dispositions de l'article 2 de la loi de 1905, qui autorise, sans l'y obliger, l'autorité compétente à rémunérer les aumôniers. Dans la mesure où le décret contesté crée une indemnité nouvelle qui se substitue à celle qui existait auparavant, «la création et la définition des conditions d'octroi d'une telle indemnité relèvent de la compétence du pouvoir réglementaire».

La compétence du pouvoir réglementaire n'étant pas en cause, encore fallait-il que son intervention ne portât pas atteinte au droit qu'ont les autorités religieuses des différents cultes de désigner en toute indépendance les personnes qualifiées pour exercer les fonctions d'aumôniers, et le cas échéant pour mettre fin à leurs fonctions. Conformément à la règle traditionnelle dite de la «double investiture», le Conseil d'État confirme que les aumôniers ne peuvent être recrutés ou agréés «que sur proposition d'une autorité représentative de leur culte». Toutefois ce principe ne fait pas obstacle, est-il précisé, à ce qu'ils soient soumis d'une part aux conditions applicables à la

16. Pour les aumôniers militaires, l'article 3 de la loi du 8 juillet 1880 sur l'aumônerie militaire, et pour les aumôniers des hôpitaux, l'article 10 de la loi du 9 janvier 1986 relative à la fonction publique hospitalière. Ces dispositions législatives renvoyant à un décret en Conseil d'État, le décret du 3 mai 2017 avait donc été adopté «le Conseil d'État entendu», en sorte qu'il faisait son retour devant la Haute juridiction, passant de la section de l'intérieur à celle du contentieux. 
catégorie d'agents publics dont ils relèvent ${ }^{17}$ «pour autant que ces conditions ne soient pas incompatibles avec leur ministère», et d'autre part «à des conditions particulières liées aux exigences propres aux services publics au sein desquels ils interviennent et aux publics auxquels ils s'adressent ».

En l'espèce, cette «condition particulière» que constitue l'exigence de diplôme « repose, indique le Conseil d'État, sur la poursuite d'objectifs d'intérêt général et de sauvegarde de l'ordre public en lien avec la mission de ces aumôniers». Les objectifs en question, tels qu'en atteste l'histoire mouvementée de la création des DU laïcité, tiennent en réalité à la volonté des pouvoirs publics de lutter contre la radicalisation islamiste, en particulier dans les prisons, à travers le recrutement d'aumôniers acquis aux valeurs de la République. Il était évidemment difficile de servir une telle réponse aux autorités religieuses catholiques à l'origine du recours, et c'est pourquoi l'arrêt énonce dans une formule euphémique et quelque peu flegmatique que les objectifs poursuivis en imposant cette formation sont « en lien avec la mission de ces aumôniers, qui interviennent dans des lieux fermés ou isolés, auprès d'agents ou de publics dont la liberté de mouvement est limitée, afin de leur permettre le libre exercice de leur culte». On a connu le juge administratif mieux inspiré dans ses explications.

Dans le cadre de ce recours structuré où toutes les fragilités du dispositif ont été successivement pointées, le Conseil d'État avait également à se prononcer sur d'éventuelles atteintes au principe de libre organisation des cultes, composante essentielle de la liberté religieuse. La réponse des juges tient ici en trois points. D'une part, la liberté qu'ont les cultes de choisir les personnes appelées à intervenir comme aumôniers dans les services publics n'est pas remise en cause par le décret. Simplement, il reviendra aux autorités religieuses de prescrire aux personnes qu'elles désigneront d'obtenir au préalable le diplôme requis. D'autre part, l'exigence d'un diplôme n'a pas «pour effet» d'encadrer l'exercice des cultes dès lors que les conditions de l'activité religieuse des aumôniers au sein des services publics ne sont en rien modifiées par le décret. Enfin, la formation imposée aux futurs aumôniers est selon les juges purement laiqque, en ce qu'elle «ne porte pas sur leur ministère religieux, mais sur l'environnement social, institutionnel et juridique dans lequel s'exerce leur activité d'aumônier et n'implique pas que l'administration, comme les enseignants y participant, porte une appréciation sur le contenu des croyances concernées ». À cela s'ajoute, même si l'arrêt ne le dit pas, que les formations pourront être suivies au sein d'établissements

17. C'est le cas pour les aumôniers contractuels dans les armées ou les hôpitaux. 
d'enseignement supérieur privés d'intérêt général à caractère confessionnel, tels que les instituts catholiques, qui pour certains ont d'ores et déjà mis en place une telle formation.

Bien qu'elle soit fondée légalement, la condition nouvelle de diplôme imposée par le décret n'en est pas moins contraignante et même potentiellement dissuasive, ce qui mérite d'être souligné compte tenu de la difficulté qu'éprouvent les religions à recruter en leur sein des volontaires pour s'investir comme aumôniers dans des activités de soutien. À cet égard, un examen rigoureux, par le Conseil d'État, de l'exigence de proportionnalité de la contrainte imposée (125 heures annuelles au minimum) au regard des objectifs poursuivis, n'aurait pas été inutile. Si la question n'est pratiquement pas abordée, c'est parce que le diplôme n'est exigé en réalité que pour accéder à un statut contractuel (militaire ou hospitalier), ou pour bénéficier d'une indemnisation publique (établissements pénitentiaires). L'absence de diplôme n'interdit pas en revanche que des aumôniers bénévoles ou rémunérés par leur culte puissent continuer à intervenir au sein des services publics, dans des conditions qui relèvent de la pratique propre à chacune des institutions concernées.

En l'absence d'obligation, pour les autorités publiques, de rémunérer les prestations d'aumônerie, l'accès d'aumôniers non rémunérés ne peut donc être empêché dès lors qu'il est une condition du libre exercice des cultes. C'est d'ailleurs la situation de ces aumôniers non diplômés, et par voie de conséquence non rémunérés, qu'avaient mise en avant les auteurs du recours, dénonçant une «différence de traitement entre des personnes susceptibles de remplir les missions d'aumônerie». L'argument est rejeté au motif qu'une telle différence de traitement est en rapport direct avec l'objet du décret (s'assurer des connaissances des aumôniers dans les domaines civil et civique) et qu'elle «n'est pas manifestement disproportionnée au regard des objectifs poursuivis par l'État». On l'a dit, cette question de la proportionnalité aurait mérité un examen plus approfondi.

De surcroît, dès lors que l'économie générale du dispositif - tout autant que sa légalité - repose sur le postulat selon lequel la possession du diplôme n'est pas une condition d'accès aux fonctions d'aumônerie, on est conduit à s'interroger sur la condition de ces aumôniers non diplômés qui continueront à intervenir, et qui aurait mérité elle aussi d'être harmonisée dans le décret pour donner sa cohérence à l'ensemble. Dans le cas d'une aumônerie fortement institutionnalisée telle que l'aumônerie militaire, les fonctions religieuses sont assurées pour l'essentiel par des aumôniers «statutaires», et le rôle des aumôniers «libres» ne peut rester que marginal. 
Si les textes relatifs à l'aumônerie ont pu voir le jour en 2017, c'est parce que les universités ont accepté de mettre en place les cursus conduisant à la délivrance du diplôme de formation civile et civique. Mais alors même que le contenu de cette formation est explicité par l'arrêté du 5 mai 2017 adopté dans le prolongement du décret, le diplôme qui en est le support est un simple diplôme d'université pour lequel la compétence revient intégralement aux universités en application de l'article L. 613-2 ${ }^{18}$ du Code de l'éducation. Le commentaire qui avait été fait du décret dans la présente revue au moment de sa publication avait souligné ce paradoxe ${ }^{19}$, et l'argument a été repris comme moyen de droit par les auteurs du recours. Mais le Conseil d'État, insensible à cette défense de l'autonomie universitaire, a considéré que l'arrêté n'a pas pour objet «de réglementer l'organisation de diplômes propres délivrés par les établissements d'enseignement supérieur, mais seulement de fixer les critères et les procédures permettant à de tels diplômes d'être inscrits sur cette liste ${ }^{20} »$. Il est vrai que l'arrêté prévoit l'établissement, par les ministères de l'Intérieur et de l'Éducation nationale, d'une liste des formations dont les diplômés pourront accéder aux fonctions d'aumôniers contractuels ou rémunérés. En ce sens la réponse du Conseil d'État est parfaitement recevable. Mais il est tout aussi vrai que l'arrêté définit le contenu minimal de la formation, ses conditions d'accès, sa durée et le principe d'une évaluation des connaissances, et que l'inscription sur la liste, après due vérification des pièces requises, a toutes les apparences d'une procédure interministérielle d'habilitation de la formation (assortie d'un soutien financier annuel du ministère de l'Intérieur) qui ne veut pas dire son nom. On ajoutera, pour terminer, que l'ambivalence de cette formation est renforcée par le renvoi express de l'arrêté aux dispositions réglementaires du Code de l'éducation relatives à la validation des acquis de l'expérience (VAE) ${ }^{21}$, et ce alors que ces dispositions ne sont d'application que pour les diplômes ou titres délivrés «au nom de l'État ${ }^{22}$ ». Les requérants ayant souligné ce point, le Conseil d'État répond en qualifiant les dispositions réglementaires du Code de l'éducation relatives à la VAE de «dispositions de droit commun», sans

18. C. éduc., art. L. 613-2: «Les établissements peuvent aussi organiser, sous leur responsabilité, des formations conduisant à des diplômes qui leur sont propres ou préparant à des examens ou des concours. [...]»

19. F. Messner, P.-H. Prélot, «Un diplôme pour l'aumônerie des services publics», Revue du droit des religions, $\mathrm{n}^{\circ} 4$, nov. 2017, p. 181-193.

20. Il s'agit de la liste des formations préparant au DU laïcité et soutenues (financièrement) à ce titre par le ministère de l'Intérieur.

21. C. éduc., art. L. 613-3.

22. Ibid. 
s'interroger sur la compétence des ministres à étendre ce dispositif général à des diplômes d'université. Autrement dit, les établissements d'enseignement supérieur, y compris ceux de caractère privé confessionnel, pourront faire bénéficier les candidats au diplôme de la validation des acquis de l'expérience, mais ils ne pourront en fixer eux-mêmes les modalités, et devront respecter les règles et les conditions définies par le Code de l'éducation pour les diplômes nationaux. Dans les faits, les universités appliquent souvent d'elles-mêmes à leurs DU les procédures législatives et réglementaires relatives à la VAE, mais avec cette différence qu'ici c'est un arrêté réglementaire qui les y contraint. L'objectif visé sur ce point par l'arrêté est de permettre au ministère de l'Intérieur d'organiser par la suite un cadrage de cette VAE pour l'ensemble des formations habilitées, afin d'éviter un contournement du dispositif par l'octroi généralisé d'équivalences.

On comprend volontiers le mécontentement des autorités religieuses catholiques, pour qui l'aumônerie n'a jamais été un simple service religieux, mais également une forme de contribution au bon fonctionnement des services publics et à l'intégration sociale des plus démunis, et qui de ce fait ont ressenti les textes de 2017 comme une forme d'humiliation. On comprend également qu'elles puissent ne pas se satisfaire complètement de la réponse que leur ont donnée un peu rapidement les juges. Mais au-delà des questions de pur droit, il reste que pour les pouvoirs publics la création des diplômes de formation civile et civique correspond à une nécessité du temps, et qu'il y a un intérêt public évident à ce que les aumôniers appelés à intervenir auprès de publics sensibles aient une connaissance avérée des institutions publiques et de la laiicité, ce que la culture commune ne permet plus malheureusement de garantir absolument. On ajoutera que depuis quelques années déjà l'Église de France fait appel à des personnels religieux originaires de l'étranger et notamment d'Afrique, d'Asie, ou encore de Pologne, pour lesquels une présentation des institutions publiques nationales et de notre système de laïcité peut être de quelque utilité. C'est à l'expérience qu'il sera possible de mesurer si le dispositif mis en place était pertinent, et s'il produit les effets attendus sur le terrain, ou bien si les contraintes imposées l'ont été inutilement. Dans l'immédiat, les textes de 2017 auront contribué paradoxalement à redonner une certaine visibilité sociale à cette institution immémoriale, mais pourtant méconnue, de l'aumônerie des institutions publiques. 